\title{
Material Processing at GHz Burst Mode by Femtosecond Laser Ablation
}

\author{
Kotaro Obata, Francesc Caballero-Lucas, and Koji Sugioka \\ RIKEN Center for Advanced Photonics, RIKEN, 2-1 Hirosawa, Wako, Saitama 351-0198 JAPAN \\ ${ }^{*}$ Corresponding author's e-mail: ksugioka@riken.jp
}

\begin{abstract}
Ablation of copper targets using a GHz burst mode with femtosecond laser pulses is investigated. To this end, the $\mathrm{GHz}$ burst mode oscillating groups of pulse train with an extremely short interval of 205 ps between each intra-pulse is generated. The pulse numbers larger than 10 in the burst significantly reduce the ablation efficiency due to the plasma shielding. The 10-pulse burst mode achieves $25 \mathrm{~mW}$ of the ablation threshold, which is four times smaller than $100 \mathrm{~mW}$ for the conventional single-pulse train femtosecond laser ablation. The $\mathrm{GHz}$ burst can perform precise ablation with smoother ablated surfaces.
\end{abstract}

DOI: $10.2961 /$ jlmn.2021.01.2004

Keywords: ablation, burst-mode, GHz ablation, femtosecond laser, micro-machining, copper, metal

\section{Introduction}

Ablation using femtosecond laser pulses makes it possible to realize precision material processing with little thermal damage for diverse applications in both scientific and industrial fields. [1] Recently, in order to explore more efficient ablation process with higher quality, the ablation using a burst mode that oscillates groups of high-repetition rate pulse train with an extremely short time interval at $\mathrm{GHz}$ has been investigated. [1-4] The burst mode femtosecond laser pulses at $\mathrm{GHz}$ enables to ablate the target material before the residual thermal energy deposited by previous pulses diffuses away from the processed area. Thus, the $\mathrm{GHz}$ burst mode ablation enables increasing the ablation efficiency by an order of magnitude while decreasing the required pulse energy by three orders of magnitude. In addition, the $\mathrm{GHz}$ burst mode ablation improves the ablation quality with suppressed thermal effect, for which it has been hypothesized that the average temperature inside the target material is reduced due to physical removal of thermal energy with the ablated materials from the ablated region to keep the target material cold (ablation cooling). Even for higher powers achieving higher processing speed, the GHz burst mode ablation can greatly reduce the thermal effect for high quality ablation of soft-tissues. [2] In the meanwhile, some other groups have reported that ablation using the $\mathrm{GHz}$ burst pulses tremendously drops the ablation efficiency as compared with conventional ablation using a single-pulse train of femtosecond laser (single-mode). [3, 5] The GHz burst mode ablation just stands at the dawn, and accumulation of data for different conditions as well as various materials is highly demanded. In this study, we investigate the ablation characteristics of copper $(\mathrm{Cu})$ by using the burst mode femtosecond laser pulses at $\mathrm{GHz}$.

\section{Experimental setup}

Figure 1 shows the schematic illustration of experimental setup for the $\mathrm{GHz}$ burst mode ablation. The ablation experiments were performed using ultrashort laser pulses

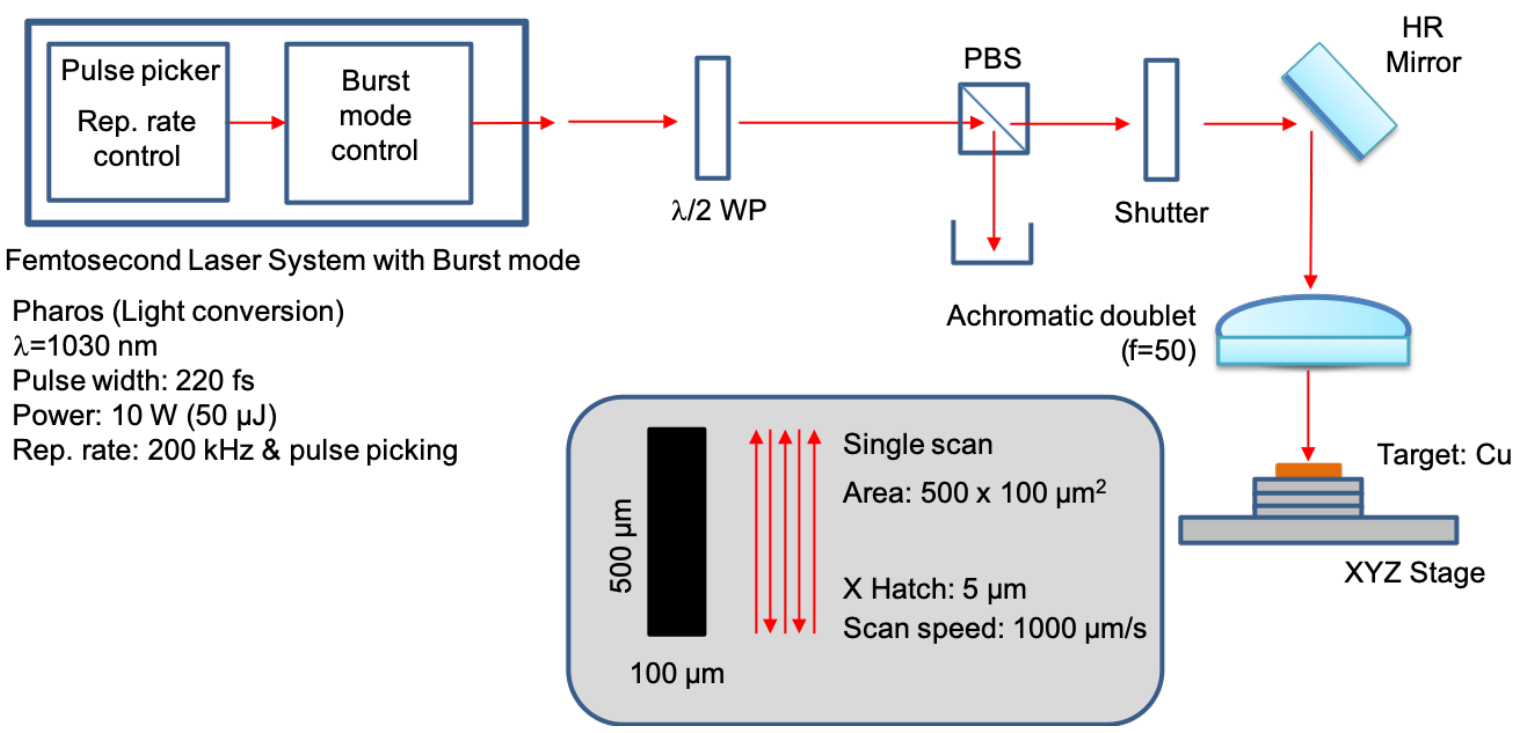

Fig. 1 Schematic illustration of experimental setup for ablation using GHz bust mode. 


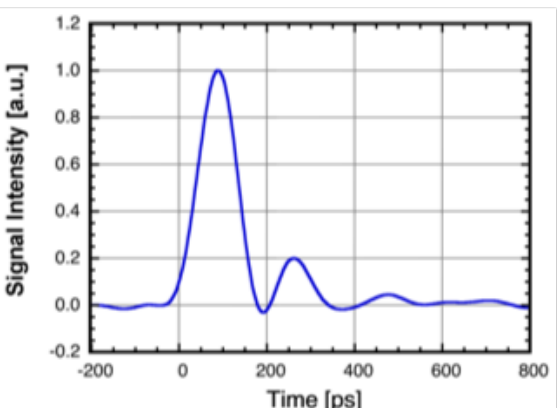

Single pulse (Original)

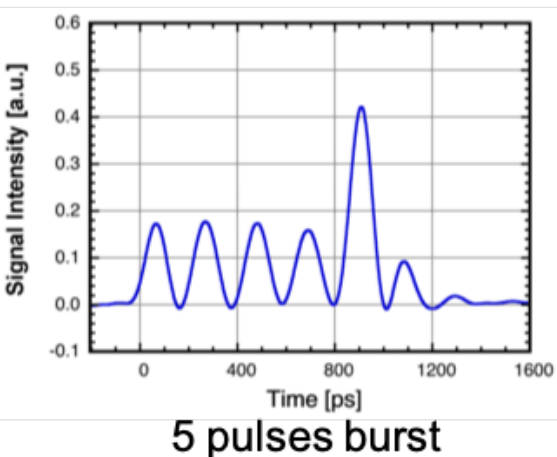

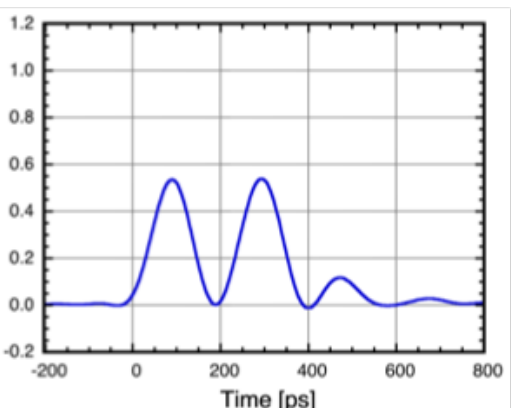

2 pulses burst

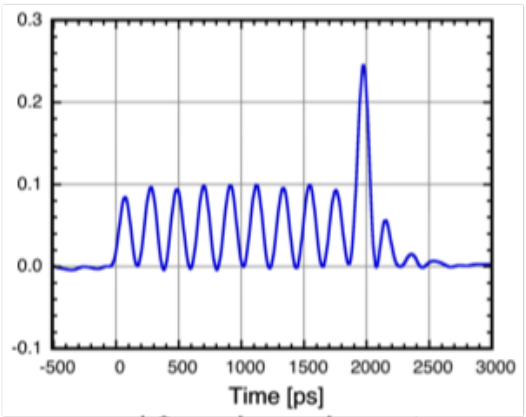

10 pulses burst

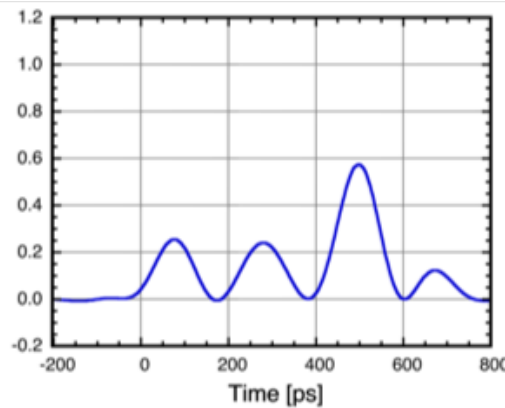

3 pulses burst

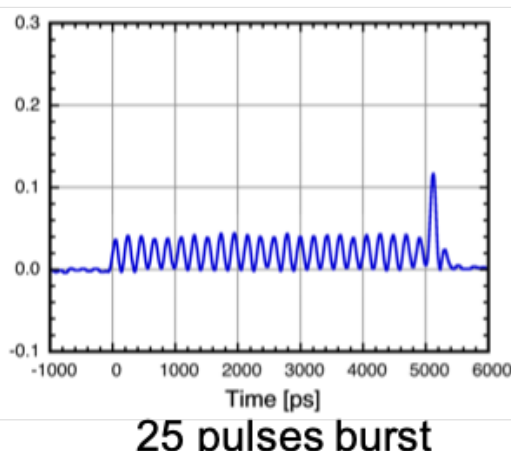

Fig. 2 Waveforms of different numbers of intra-pulses in the burst.

obtained from a $\mathrm{Yb}: \mathrm{KGW}$ based high-power femtosecond laser system with a central wavelength of $1030 \mathrm{~nm}$ and a pulse duration of $220 \mathrm{fs}$ (Light conversion Ltd., Pharos). The femtosecond laser system enables to generate $\mathrm{GHz}$ burst pulses with the same wavelength of $1030 \mathrm{~nm}$. The number of pulses in each burst can be adjusted up to 25. Figure 2 shows the measured waveforms of the original single mode operation and $\mathrm{GHz}$ burst mode operation for different intrapulse numbers. The burst pulse trains with a constant intrapulse interval of 205 ps (frequency $4.88 \mathrm{GHz}$ ) were generated. The energy of intra-pulse was almost constant, but for the pulse numbers larger than 5, the last pulse was much more intense due to characteristics of the opto-mechanical configuration of laser system used in which the burst pulse trains were generated. Additionally, negligibly small pulse was generated at the end. Duration of each intra-pulse remained constant at $220 \mathrm{fs}$. The pulse energy was adjusted by using the polarizing optics composed of a $\lambda / 2$ waveplate and a polarizing cubic beam splitter, and the number of irradiation pulses was controlled by a mechanical shutter. The attenuated laser pulses were focused to a $19.2 \mu \mathrm{m}$ spot in diameter by an achromatic lens with a focal length of $50 \mathrm{~mm}$. Oxygen-free $\mathrm{Cu}$ plates were used for the sample. The sample was set on the computer controlled XYZ stage (OptSigma, OSMS20-85) to scan the laser beam on it with a constant operating speed of $1000 \mu \mathrm{m} / \mathrm{s}$. The reverse scan was employed with a pitch interval of $5 \mu \mathrm{m}$ to ablate $1000 \times 500$ $\mu \mathrm{m}^{2}$ area. After the ablation process, depth of ablated area and surface morphology were evaluated by a laser scanning microscope (Zeta Instruments Inc, Zeta-20) and a scanning electron microscope (SEM) (Thermo Fisher Scientific K.K., Quattro S), respectively.

\section{Results and discussion}

Figure 3 (a) shows dependence of ablation depth on the input laser power (lower horizontal axis) and the burst laser fluence (upper horizontal axis) for the burst mode with different intra-pulse numbers (up to 25 pulses) and the singlemode femtosecond laser ablation. The burst laser fluence was calculated by dividing the input laser power by the product of repletion rate and the irradiation area. In this experiment, each burst with different intra-pulse numbers and the single pulse were repeated at $200 \mathrm{kHz}$, so that the intra-pulse energy was equal to the pulse energy of single mode divided by the intra-pulse number. The ablation performed by the burst mode shows much shallower ablation depth compared with the single mode ablation at the same input laser power and the burst laser fluence, which means that the ablation efficiency of $\mathrm{GHz}$ burst is lower than the single mode. Furthermore, the depth significantly decreases for the intrapulse numbers larger than 10 .

The reason for the decrease of depth at larger number of intra-pulses shown in Fig. 3(a) can be attributed to plasma shielding as shown in Fig. 4. Specifically, the laser induced plasma is typically generated and shields the incident laser beam at the time scale of $10 \mathrm{ps} \sim$ several ns after laser irradiation, which depends on materials and irradiation conditions. [6, 7] In particular, in case of $\mathrm{Cu}$ ablation, the plasma shielding effect starts from $10-50 \mathrm{ps}$, and is then maximized at around 1ns. [7] Considering the intra-pulse interval of 205 ps, the effect of plasma shielding gradually increases until the $5^{\text {th }}$ intra-pulse arrives. After approx. 1ns, the plasma shielding effect is saturated. The $5^{\text {th }}$ and higher order intra pulse arrive at the target at $1 \mathrm{~ns}$ or later after the $1^{\text {st }}$ intra- 


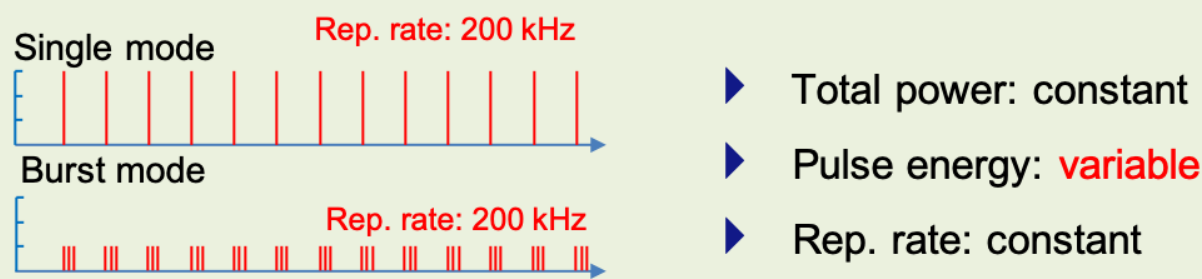

(a)

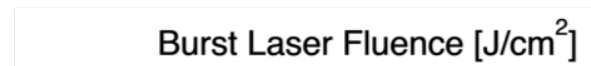

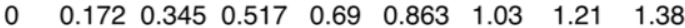

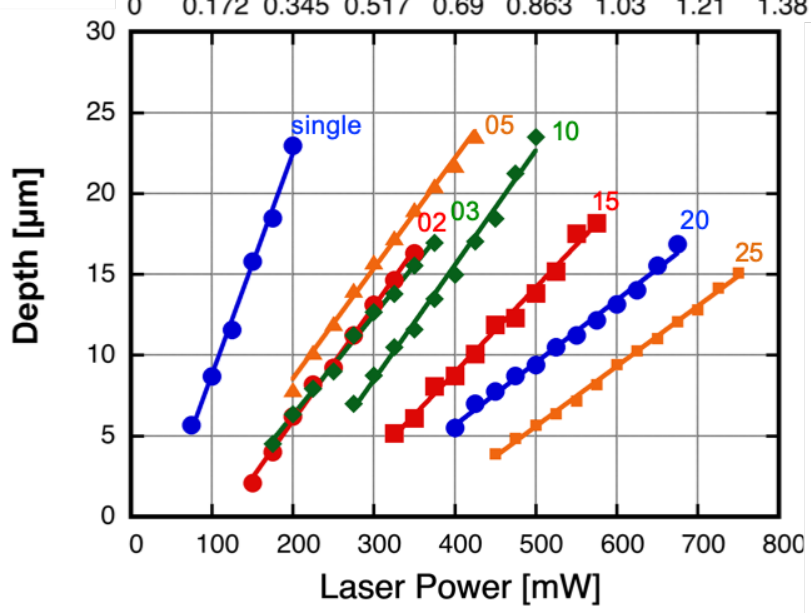

(b)

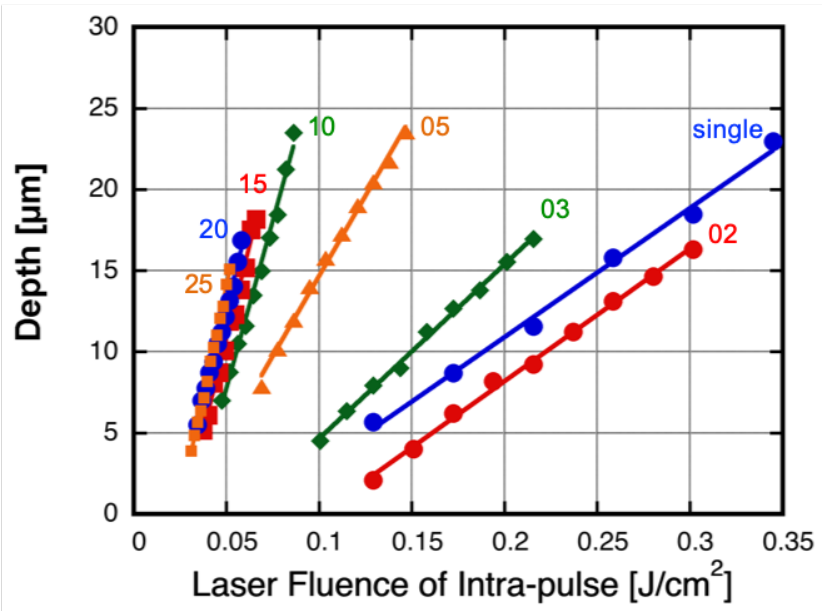

Fig. 3 Dependence of ablation depth on the input laser power (lower horizontal axis) and the burst laser fluence (upper horizontal axis) (a), and average laser fluence of intra-pulse (b) for the burst mode with different intrapulse numbers and the single-mode femtosecond laser ablation.

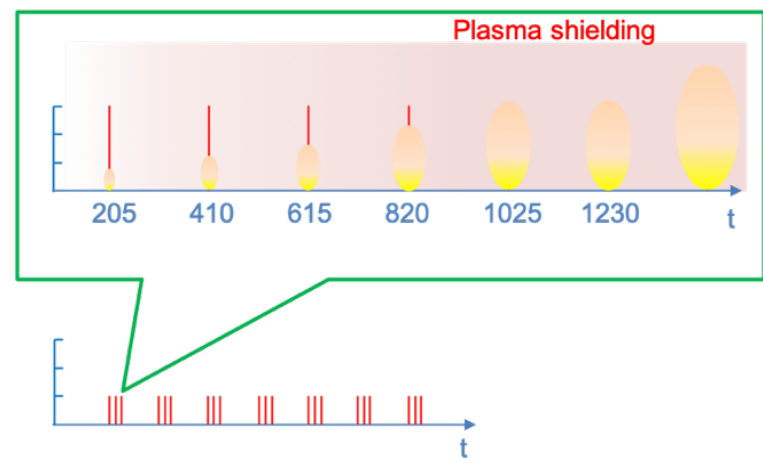

Number of Burst Plasma shielding

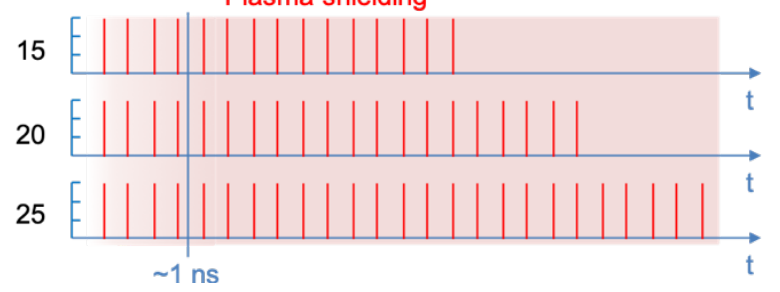

Fig. 4 Schematic illustration of influence of the plasma shielding on ablation.

pulse irradiation. Therefore, those intra-pulses in the burst should be shielded by the plasma so as not to contribute ablation, resulting in reduction of the ablation efficiency. On the other hand, variations of ablation depth on laser power show linear dependence even at the higher laser power in spite of the fact that the plasma shielding effect is stronger for the higher laser power which should lead to a deviation from the linear dependence. This result may give two possible reasons for reduction of the ablation efficiency by the burst mode ablation, in particular, with larger intra-pulse numbers, which conflict with each other. First one is the losses of deposited energy through heat dissipation. However, the results shown in Fig. 5 does not support the heat dissipation as discussed below. Ilday et al. have reported that highly repetitive laser irradiation in the $\mathrm{GHz}$ range ablation carries away thermal energy along with the generated ablation particles (ablation cooling). [2] The thermal energy carried away by the ablation cooling may be the other possible reason for the reduction of ablation efficiency and decease of ablation depth.

Meanwhile, Fig. 3(b) shows the dependence of ablation depth on the average laser fluence of intra-pulse for different intra-pulse numbers. The burst mode with the intra-pulse numbers larger than 5 significantly reduces the ablation threshold in the laser fluence of intra-pulse, while the slope of lines for dependence of depth and laser fluence becomes much larger. This result might suggest that the subsequent pulses in the burst could successively induce ablation before the heat generated by the preceding pulses diffused away from the processed regions. Almost the same dependency for the intra-pulse numbers larger than 5 should be due to the plasma shielding as described above. On the other hand, shallower depths for the intra-pulse number of 2 as compared with the single mode at the same fluence and almost the same slope of lines require further investigation. 

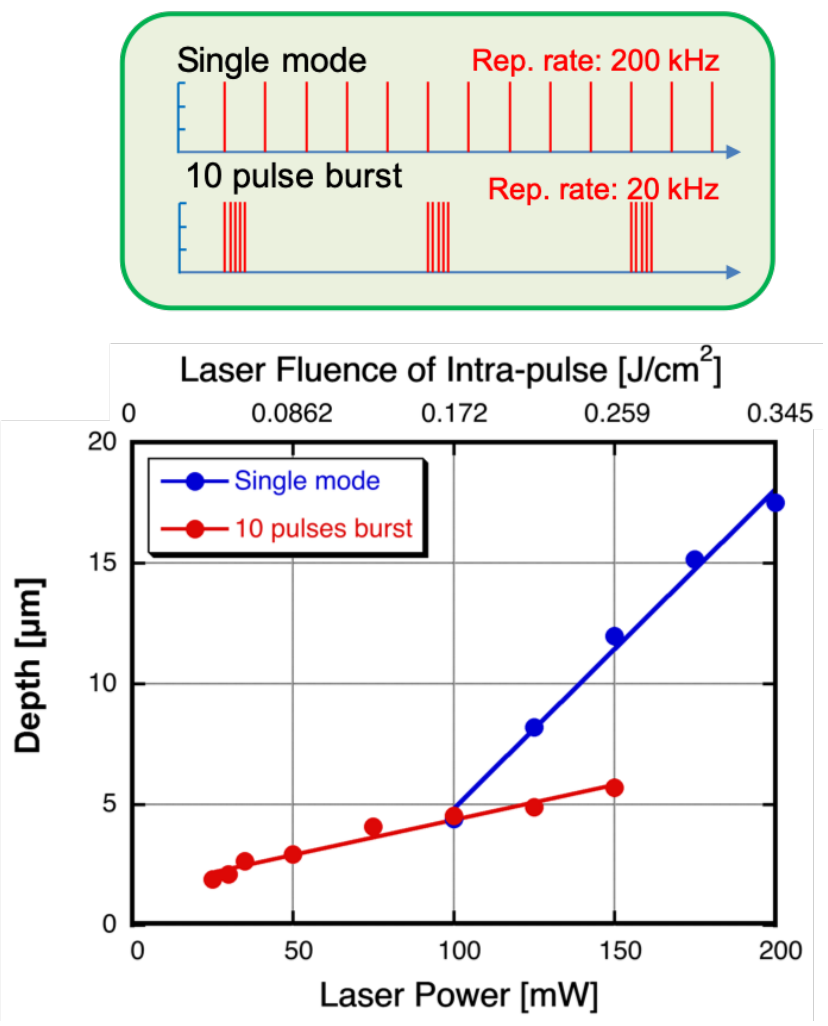

Fig. 5 Dependence of ablation depth on the input laser power (lower horizontal axis) and average laser fluence of intra-pulse (upper horizontal axis) for the 10 burst pulse trains (20 kHz rep. rate) and single-mode femtosecond laser ablation ( $200 \mathrm{kHz}$ rep. rate).

Figure 5 shows dependence of ablation depth on the input laser power (lower horizontal axis) and the average laser fluence of the intra-pulse (upper horizontal axis) for femtosecond laser ablation by the 10-pulse burst mode and the single mode. In this experiment, pulse energy of the single mode and the intra-pulse was kept constant to make the input laser power same. Therefore, the repetition rate of the burst was set at $20 \mathrm{kHz}$, while the single mode, $200 \mathrm{kHz}$ to make the total input energy same.

Ablation didn't take place at the power below $25 \mathrm{~mW}$ $\left(43.1 \mathrm{~mJ} / \mathrm{cm}^{2}\right)$ and $100 \mathrm{~mW}\left(172 \mathrm{~mJ} / \mathrm{cm}^{2}\right)$ for the burst mode and the single mode, respectively. Therefore, the burst mode can reduce the ablation threshold to four times smaller as compared with the single-mode ablation. The great decrease of the ablation threshold can be deduced that the burst mode ablation in the $\mathrm{GHz}$ range suppresses the heat dissipation and enhances the laser ablation at the localized volume. Meanwhile, the slope of line for dependence of ablation depth on laser power for the burst mode is significantly smaller than that for the single mode. Additionally, the ablation depth for the burst mode at $150 \mathrm{~mW}\left(259 \mathrm{~mJ} / \mathrm{cm}^{2}\right)$ is less than half of those for the single mode. The larger ablation depth by the single mode may be due to larger heat penetration resulting in larger thermal effect. Thus, the burst mode ablation can perform the precise ablation, although it reduces the ablation efficiency.

The surface morphology of ablated areas was observed by SEM. Figure 6 shows the SEM images of the samples fabricated by the single mode (a) and the burst mode with 2
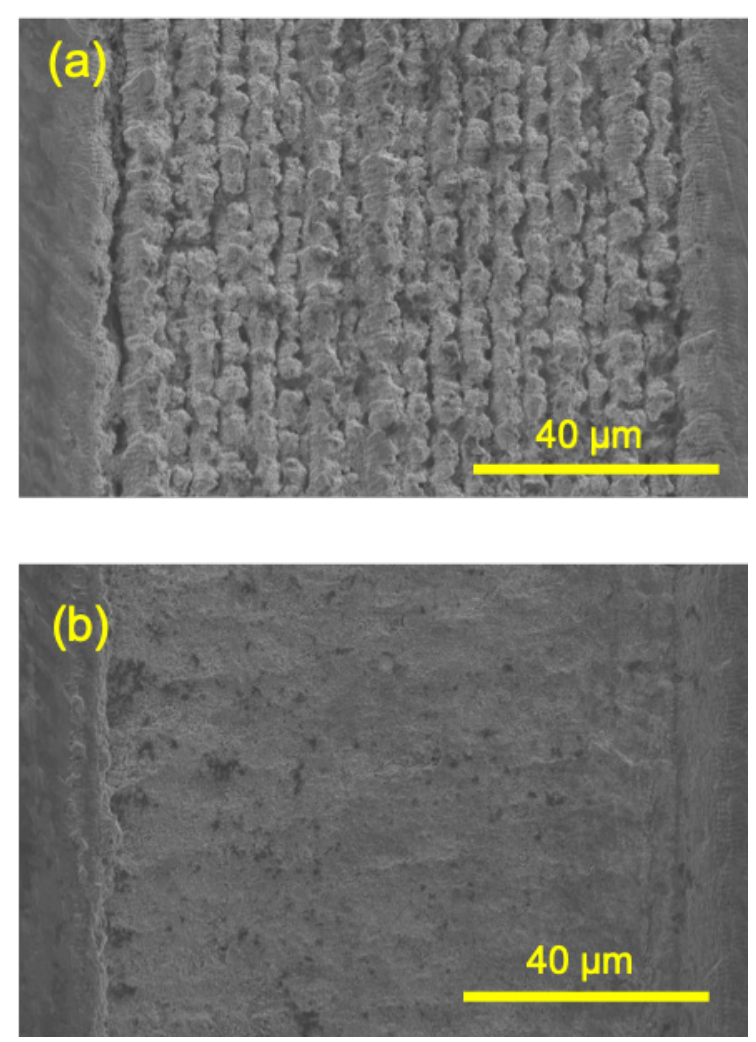

Fig. 6 The SEM images of the samples fabricated by the single mode (a) and the burst mode with 2 intrapulses (b). Samples used for this observation corresponded to the samples with the ablation depth of approx. $10 \mu \mathrm{m}$ in Fig. 3 .

intra-pulses (b). Samples used for this observation corresponded to the samples with the ablation depth of approx. 10 $\mu \mathrm{m}$ in Fig. 3. The single-mode laser ablation produced the line shape grooves which were parallel to the laser scanning direction. The ablated surface is significantly roughened as compared with the virgin substrate. In contrast, the burst mode sample shows relatively smooth surface as if reflow of shallow melted layer by thermal effects. The mechanism of improvement of surface roughness by the burst mode is still unclear. Assuming the ablation cooling hypothesized by Ilday et al. [2], the GHz ablation should lead to low thermal damage and reduction of formation of the heat affected zone (HAZ). The results shown in Fig. 5, specifically less heat penetration by the $\mathrm{GHz}$ burst, may support this hypothesis. However, the reflow of shallow melted layer due to heat accumulation in multi-pulse regime as described above is considered to be another possible mechanism. At least, the precise ablation with low ablation rate by the $\mathrm{GHz}$ burst should contribute to achieve smoother ablated surface. Consequently, the GHz burst ablation is beneficial to create ablated surface with better quality.

\section{Conclusions}

We have demonstrated ablation of $\mathrm{Cu}$ targets with the femtosecond burst mode laser system. Ablation characteristics by the burst pulse trains composed of different number of intra-pulses with $\mathrm{GHz}$ repetition rate were investigated. It was found that the burst mode ablation lowered ablation efficiency as compared with the conventional single-mode 
femtosecond laser ablation. The ablation efficiency decreased with increasing the number of intra-pulses due to the plasma shielding. The laser induced plasma was expected to be formed at about $2 \mathrm{~ns}$ after the $1^{\text {st }}$ pulse irradiation, so that $10^{\text {th }}$ and later pulses in the burst could not reach the surface of the sample, which prohibited those pulses from contributing the ablation and thereby reduced the ablation efficiency. On the other hand, the GHz burst mode with 10 intrapulses reduced the ablation threshold down to four times difference as compared with the conventional single-mode femtosecond laser ablation. More importantly, the $\mathrm{GHz}$ burst was able to improve the quality of ablated surfaces. Overall, the $\mathrm{GHz}$ burst pulse generation and its use for laser ablation will offer significant applications.

\section{Acknowledgments and Appendixes}

This work was partially supported by MEXT Quantum Leap Flagship Program (MEXT Q-LEAP) Grant Number JPMXS0118067246. This work was partially supported by a grant from the Amada Foundation (AF-2019224-B3).
[1] K. Sugioka and Y. Cheng: Light Sci. Appl., 3, (2014) e149.

[2] C. Kerse, H. Kalaycıŏ̆lu, P. Elahi, B. Çetin, D. K. Kesim, Ö. Akçaalan, S. Yavaş, M. D. Așık, B. Öktem, H. Hoogland, R. Holzwarth, and F. Ö. Ilday: Nature, 537, (2016) 84.

[3] X. Wolters, G. Bonamis, K. Mishchick, E. Audouard, C. Hönninger, and E. Mottay: Procedia Manufacturing, 36, (2019) 200.

[4] K. Mishchik, G. Bonamis, J. Qiao, J. Lopez, E. Audouard, E. Mottay, C. Hönninger, and I. Manek-Hönninger: Opt. Lett., 44, (2019) 2193.

[5] T. Hirsiger, M.Gafner, S. Remund, M. V. Chaja, A. Urniezius, S. Butkus, and B. Neuenschwander: Proc. SPIE, Vol. 11267, (2020) 112670.

[6] S. S. Mao, X. Mao, R. Greif, and R. E. Russo: Appl. Phys. Lett. 77, (2000) 2464.

[7] D. J. Förster, S. Faas, S. Gröninger, F. Bauer, A. Michalowski, R. Weber, and T. Graf: Appl. Sur. Sci., 440, (2018) 926.

\section{References}

(Received: July 16, 2020, Accepted: January 3, 2021) 